\title{
Política institucional en la UNAN-Managua para la adaptación y mitigación al cambio climático
}

Delia Moreno $^{1}$

\section{RESUMEN}

El Cambio Climático es una de las problemáticas que enfrenta Nicaragua, cuya política de atención está sustentada en la Estrategia Nacional de adaptación al cambio climático 2010-2015 y retomada en el artículo 60 de

la Constitución Política del país, referida en el artículo 60 de la misma, y el Plan Nacional de Desarrollo Humano. Ambas coinciden que la deforestación de grandes extensiones del territorio, ha provocado la erosión del suelo y el aumento de zonas secas poniendo en riesgo la seguridad alimentaria de la población, la economía, el comercio y la biodiversidad. Este artículo es una reflexión sobre el rol social de la universidad en la formación de los profesionales con una amplia visión y sensibilización para la adaptación y mitigación al cambio climático, papel que puede ser fortalecido y de impacto a través del establecimiento de una política institucional en la Universidad Nacional Autónoma de Nicaragua, Managua, como instrumento que facilite un accionar integral, tomando en cuenta los recursos potenciales de todos los actores que convergen en esta alma mater, que de manera articulada permitan la coordinación y disminución de los impactos adversos del cambio climático, y ofrecer un marco de acciones que contribuyan a incrementar la resiliencia ambiental.

Palabras clave: política institucional, adaptación al cambio climático, mitigación al cambio climático.

Recibido: 28 de junio de 2017

Aceptado: 09 de noviembre de 2017

1 Docente UNAN-Managua FAREM-Estelí. Estudiante del Doctorado Gestión de la Calidad de la Investigación. UNAN-Managua FAREM-Estelí. Correo electrónico: delmorenoc@yahoo.com 


\title{
UNAN-Managua Institutional policy for adaptation and mitigation for climate change
}

\begin{abstract}
Climate Change is one of the problems faced in Nicaragua, whose policy of attention is based on the National Strategy for Adaptation to Climate Change 2010-2015 and taken up in Article 60 of the Political Constitution of the country, referred to in Article 60 of the same, and the National Plan of Human Development. Both agree that the deforestation of large areas of the territory has led to soil erosion and the increase of dry areas, putting at risk the food security of the population, the economy, trade and biodiversity. This article is a reflection on the social role of the university in the training of professionals with a broad vision and awareness for adaptation and mitigation to climate change, a role that can be strengthened and impact through the establishment of an institutional policy in the National Autonomous University of Nicaragua, Managua, as an instrument that facilitates an integral action, taking into account the potential resources of all the actors that converge in this alma mater, which in an articulated manner allow the coordination and reduction of the adverse impacts of climate change, and offer a framework of actions that contribute to increasing environmental resilience.
\end{abstract}

Keywords: institutional policy, adaptation to climate change, mitigation to climate change. 


\section{INTRODUCCIÓN}

Las variaciones del clima se han convertido en un gran desafío para todos los seres humanos ya que afecta la economía, el comercio, la seguridad, y que dominará las políticas mundiales y nacionales a medida que se hagan más evidentes los efectos. Estos son inevitables y van desde la elevación, y el calentamiento del mar hasta el derretimiento de los casquetes polares y los glaciares; así como mayor incidencia de las inundaciones, sequías y pérdida de los sistemas naturales; poniendo en un alto riesgo la vida en el planeta.

Según (UNFCCC, 2001), Unidos por el Clima en su informe refiere que "En 1988, la Asamblea General de las Naciones Unidas aprobó la resolución 43/53, propuesta por el Gobierno de Malta, en la que se pedía “... la protección del clima para las generaciones actuales y futuras de la humanidad". Durante el mismo año, los órganos rectores de la Organización Meteorológica Mundial y del Programa de las Naciones Unidas para el Medio Ambiente crearon un nuevo organismo, el Grupo Intergubernamental de Expertos sobre el Cambio Climático (IPCC), para orientar y evaluar la información científica sobre este tema"

Desde su formación el IPCC, presenta informes cada cinco años sobre la situación de los conocimientos científicos relacionados con el cambio climático y prepara documentos técnicos sobre aspectos concretos en respuesta a las peticiones de la Conferencia de las Partes (CP o COP) es el máximo órgano responsable de la toma de decisiones en las reuniones de la Convención de las partes (CP). La Convención estableció dos órganos subsidiarios permanentes de apoyo en el Asesoramiento Científico y Tecnológico (OSACT) y la (OSE) como Órgano Subsidiario de Ejecución para evaluar y examinar la ejecución de las decisiones, aspectos financieros y seguimiento a los compromisos adquiridos por los países en cuestiones de emisiones (UNFCCC, 2017).
El cambio climático se convirtió en un problema central para la comunidad científica internacional en la década de los años 70 del siglo pasado, dando origen en 1979 a la I Conferencia Mundial sobre el Clima. En la arena política, los esfuerzos por prevenir y minimizar su impacto sobre la Humanidad cristalizaron en la Convención Marco de las Naciones Unidas (CMNUCC) efectuada en 1992 materializando el Protocolo de Kyoto.

Este Protocolo contiene el compromiso de los países industrializados a estabilizar las emisiones de gases de efecto invernadero (GEI). La Convención da seguimiento al cumplimiento del compromiso que asumen los 37 países industrializados y la Unión Europea para reducir los GEI, reconociendo según (UNFCC, s.f.) "son los principales responsables de los elevados niveles de emisiones de GEI que hay actualmente en la atmósfera, y que son el resultado de quemar fósiles combustibles durante más de 150 años. En este sentido el Protocolo tiene un principio central: el de la «responsabilidad común pero diferenciada».

UNFCC también plantea que el Protocolo ha movido a los gobiernos a establecer leyes y políticas para cumplir sus compromisos, a las empresas a tener el medio ambiente en cuenta a la hora de tomar decisiones sobre sus inversiones, y además ha propiciado la creación del mercado del carbono. Este Protocolo es considerado como primer paso importante hacia un régimen verdaderamente mundial de reducción y estabilización de las emisiones de GEI, y proporciona la arquitectura esencial para cualquier acuerdo internacional sobre el cambio climático que se firme en el futuro. Presentando informes desde 1990 en los cuales confirman científicamente la amenaza que representa el cambio climático para la humanidad.

En la segunda Conferencia Mundial sobre el Clima celebrada en Ginebra más tarde ese mismo año se pidió la creación de un tratado mundial. La Asamblea General respondió aprobando la resolución 45/212, en la que se ponían oficialmente en marcha negociaciones 
acerca de una convención sobre el cambio climático, bajo la dirección del Comité Intergubernamental de Negociación (CIN).

El cuarto informe del (IPCC, 2007), refiere que "sobre el calentamiento global ocasionado por los gases de efecto invernadero generados por actividades antrópicas, existe una preocupación por los actuales gobernantes quienes en las diferentes cumbres han debatido su compromiso político". Lo que pone en evidencia que para alcanzar una política de respuesta global y efectiva es sumamente necesaria la comprensión de la multicausalidad centrado en el modo de producción y consumo, sumado a la dinámica de los sistemas naturales como parte del problema.

En este sentido el cambio climático constituye el mayor reto que ha enfrentado y continuará enfrentando el planeta y los seres humanos en el futuro. Existe un debate en cuanto a la génesis ya que por un lado están los procesos naturales (emisiones de $\mathrm{CO} 2$ de los océanos y/o los ciclos de carbono) y por otra parte las actividades antropogénicas (las emisiones de los gases invernaderos. (Lugo Marín, 2015, pág. 6). Estos gases de efecto invernadero (GEI), son de origen natural y antropogénico, están conformados por el vapor de agua (H2O), el dióxido de carbono (CO2), el dióxido de nitrógeno (NO2), el metano (CH4) y el Ozono (O3). Los gases incorporados a la atmósfera producidos por los seres humanos los clorofluorocarbono (CFC), hidroflurocarbono (HFC) y hexafluoruro de azufre (SF6).

El cambio climático se manifiesta en un calentamiento global ocasionado por el efecto invernadero. Estos gases se han incrementado considerablemente en la atmósfera lo que ha propiciado una rápida retención del calor del planeta. Con base en este planteamiento la dinámica de los sistemas naturales tiene un papel importante pero las acciones antropogénicas probablemente han actuado como catalizadores aumentando el efecto de la dinámica del clima.
La variación de las concentraciones de gases de efecto invernadero (GEI) y aerosoles en la atmósfera, y las variaciones de la cubierta terrestre y de la radiación solar, alteran el equilibrio energético del sistema climático y que las emisiones mundiales de GEI por efecto de actividades humanas han aumentado, desde la era preindustrial, en un 70\% entre 1970 y 2004 . (IPCC, 2007).

De igual manera en el Informe Especial del IPCC sobre escenarios de emisiones (IPCC, 2000) proyecta un aumento de las emisiones mundiales de GEI de entre $25 \%$ y $90 \%$ (CO2 -eq) entre 2000 y 2030), suponiendo que los combustibles de origen fósil mantengan su posición dominante en el conjunto mundial de fuentes de energía hasta 2030 como mínimo. Otros escenarios más recientes, que no contemplan medidas de mitigación de las emisiones adicionales, arrojan resultados similares.

En ese contexto se han realizado múltiples esfuerzos en la construcción de directrices políticas a nivel global que mitigue y controle las emisiones de gases invernaderos, son ejemplos; las reuniones anuales de las Partes de la Convención Marco de Naciones Unidas sobre Cambio Climático y del Protocolo de Kyoto.

Desde lo local, también se ha trabajado en desarrollar las capacidades adaptativas con base al conocimiento local y por la otra, reflexionar en nuestro accionar individual y colectivo, en términos de ajustar nuestro sistema de valores para así poder construir modelos de mitigación y adaptación encaminados a la sustentabilidad.

Sin embargo, las problemáticas ambientales y sociales como el cambio climático, requieren de un análisis crítico, capaz de relacionarlas en todas sus dimensiones tanto para entenderlas como para atenderlas de manera eficaz, lo cual requiere la articulación de diversas disciplinas que facilite la comprensión en todas sus dimensiones, aunado al incremento de la resiliencia ambiental a todos los ámbitos y en este caso es urgente formular políticas institucionales que 
faciliten un accionar que cohesione los esfuerzos de todas las facultades en pro de la adaptación al cambio climático fortaleciendo el rol de sus ejes de trabajo: la investigación, la docencia, la extensión y la internacionalización.

La Universidad Nacional Autónoma de Nicaragua, Managua (UNAN Managua), en su misión se propone "Formar profesionales y técnicos integrales desde y con una concepción científica y humanista del mundo, capaces de interpretar los fenómenos sociales y naturales con un sentido crítico, reflexivo y propositivo, para que contribuyan al desarrollo social, por medio de un modelo educativo centrado en las persona..." (UNAN, 2016), que reafirma el compromiso social en la formación de profesionales comprometidos con las necesidades de la sociedad y su problemática.

En el contexto actual, la primer tarea de la universidad en el campo de la investigación, implica conocer que es lo que está sucediendo en el mundo con el cambio climático a partir de la implementación de los residuos fósiles tales como el carbón, gas, el petróleo; las emisiones de gases de efecto invernadero que emiten anualmente los países y sus impactos en la vida del planeta y la huella de carbono en el mundo.

Estos procesos guiados científicamente pueden contribuir significativamente a que en nuestro país se trabaje con información fiable y concreta que aporte a la toma de decisiones basadas en análisis de la realidad contribuyendo al desarrollo de la Estrategia Nacional de Cambio Climático y el Plan Nacional de Desarrollo a las particularidades de cada territorio.

La segunda tarea de las universidades está relacionada a la formación y educación que implica formar profesionales con capacidades sobre las causas y efectos del cambio climático que les permita actuar con responsabilidad en todos los ámbitos laborales y personales para incidir significativamente en los procesos productivos, políticos, tecnológicos $\mathrm{y}$ normativos.
En ese sentido, la formación de los profesionales con una visión de responsabilidad ante el cambio climático implica contribuir a la creación de instrumentos técnicos y políticos para mitigar los efectos negativos ante el cambio climático; así como construir alternativas de adaptación y superar los impactos en los espacios institucionales que ellos y ellas se desempeñen.

Con base en estos planteamientos, es sumamente necesaria contar con una política institucional para la adaptación al cambio climático que fortalecería el rol de la UNAN Managua en su contribución como generadora de información científica a la base de datos de cambio climático del país, crear capacidades en materia ambiental en la formación docente y de los profesionales; así como hacer incidencia en que la transformación curricular integre el eje transversal de cambio climático en las diversas carreras que oferta que facilite los procesos de adaptación y mitigación ante el cambio climático.

Al respecto, (Milán, 2016), manifiesta "que para enfrentar el cambio climático es necesario que desde las universidades se contribuya a elevar la productividad, mejorar la investigación y transferencia tecnológica, así como la creación de capacidades". De igual manera "es necesaria también la mitigación, con el fin de reducir los gases invernaderos, del cual, Nicaragua emite solo el 0.03 , en relación a los demás países de la región".

A nivel local en la UNAN Managua, se ha desarrollado importantes procesos y proyectos en el tema de cambio climático con recursos propios y de cooperación internacional, y es un referente para la ejecución de procesos con otras entidades con quienes ha establecido sinergias desde todos los niveles. Sin embargo estos esfuerzos requieren de cohesionar más las acciones para identificar los escenarios climáticos desde cada facultad a un nivel más concreto que permitan definir de manera concreta el rol desde cada territorio para aportar a la comunidad con datos científicos que faciliten la toma de decisiones en el desarrollo de las Estrategias 
Territoriales de Adaptación al Cambio Climático y el Plan Nacional de Desarrollo en su expresión local.

En la medida que el accionar de la UNAN Managua cuente con una política institucional facilitará incidir de manera importante en la participación de la comunidad universitaria, las entidades nacionales y locales, y la población para la adaptación al cambio climático. Esto conlleva a realizar un análisis de los recursos humanos y económicos con los que se cuentan para construir un escenario de actuación nacional y territorial.

\section{MÉTODO}

Para la elaboración de este documento, se utilizó la metodología del estado del arte, que como modalidad de investigación documental permite según, (Londoño, 2014, pág. 4) "compartir la información, generar una demanda de conocimiento y establecer comparaciones conotros conocimientos paralelos, ofreciendo diferentes posibilidades de comprensión del problema tratado o por tratar, debido a que posibilita múltiples alternativas en torno al estudio de un tema". De tal manera que se realizó una revisión y análisis documental sobre el cambio climático en el marco del estudio de doctorado Gestión de la Calidad de la Investigación Científica, desarrollada en la UNAN Managua/FAREM Estelí.

Londoño reafirma, que esta metodología es "seguirle las huellas a un proceso y un artículo del estado del arte resume y organiza los avances del conocimiento en una forma novedosa y apoya la comprensión de un campo específico de conocimiento. En consecuencia, un estado del arte estudia una porción substancial de la literatura y fuentes relevantes de información en un área y desarrolla un proceso de comprensión que converge en una visión global e integradora y en una comunicación de este resultado para otros."

De tal manera que el estado del arte es una recopilación crítica de diversas fuentes de textos de información de un área o disciplina, que de manera escrita propone el proceso cognitivo de una investigación a través de la lectura de la bibliografía encontrada durante el proceso. Es una recopilación crítica de diversos tipos de texto de un área o disciplina, que de manera escrita, formaliza el proceso cognitivo de una investigación a través de la lectura de la bibliografía hallada durante la indagación de los problemas y el contexto.

En la elaboración del artículo se hizo búsqueda de la información existente sobre el cambio climático, que permitiera comprender la problemática global, el abordaje desde diferentes puntos de vistas, las negociaciones climáticas, el contexto nacional y local sobre la adaptación al cambio climático; tomando como base la Estrategia Nacional y el Plan Nacional de Desarrollo como documentos rectores que recogen la problemática actual. Y que (Martinez, 1999) dice que "al confluir todas estas miradas, estructuran un tejido nuevo de sentidos y significados, surgido de la reflexión crítica y que permite hacer nuevas comprensiones del objeto de estudio" (p. 13).

La reflexión y análisis documental se realizó basado en los siguientes aspectos:

1. Marco global del contexto del cambio climático

2. Campos de indagación definidos y reconocidos relacionados con el tema de las políticas institucionales del cambio climático

3. Conceptos esenciales se evidencian en los documentos seleccionados para construir el estado del arte.

4. Contenidos, tópicos o dimensiones definidos en el tema de cambio climático.

5. Las políticas institucionales en el marco de la adaptación al cambio climático.

El proceso partió de recopilar la mayor información posible y pertinente sobre el cambio climático y consignar dicha información en fichas de análisis, lo que permitió estudiar la bibliografía seleccionada para tener una mayor comprensión del tema que se está estudiando. En dichas fichas se anotaron los aspectos básicos y unificadores, para luego pasar a su interpretación y elaborar el artículo científico. 
Este proceso se podría graficar de la siguiente manera:
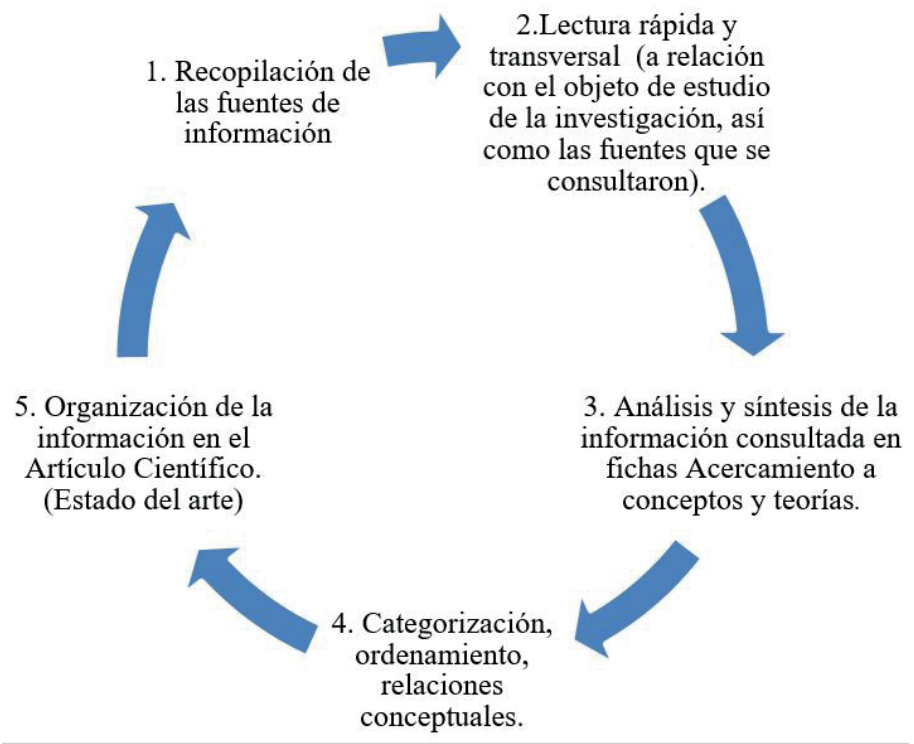

Fuente propia: basado en análisis de (Londoño, 2014).

Descripción del proceso:

a. Recopilación de las fuentes de información: consistió en realizar búsqueda en informes, documentos de internet, artículos y libros.

b. Lectura rápida y transversal de las fuentes de información (a relación con el objeto de estudio de la investigación, así como las fuentes que se consultaron. Esta revisión documental es la que permite un acercamiento a conceptos, teorías, metodologías y perspectivas, que sirven como base al artículo científico.

c. Se elaboraron fichas que permitan sintetizar las ideas centrales de cada lectura analizada.

d. Categorización, ordenamiento y relaciones conceptuales y establecer una relación precisa de las categorías (indicadores) que se van reseñando.

e. Organización del artículo científico (presentación del estado del arte).

\section{RESULTADOS DEL ANÁLISIS}

\section{Marco global del contexto del cambio climático}

Se entiende por cambio climático global al cambio de clima atribuido a la dinámica de los sistemas naturales y a la actividad humana que altera la composición de la atmósfera de la tierra y que se suma a la variabilidad natural del clima observada durante períodos de tiempo comparables.

En estudios realizados en núcleos de hielo (OMM, 2009), se ha establecido una sólida correlación entre el contenido de $\mathrm{CO} 2$ en la atmósfera y la temperatura terrestre; altas concentraciones atmosféricas de este gas han coincidido con incrementos en la temperatura media global. En la actualidad, finalmente, los impactos tienen dimensión global, pues, o afectan a bienes ambientales públicos globales, como la atmósfera en el cambio climático o el adelgazamiento de la capa de ozono, o tienen escala global, como en lo que concierne a la pérdida de biodiversidad.

La (OMM, 2009), refiere que la principal causa del cambio climático global es el efecto invernadero que se produce cuando la superficie terrestre, los océanos y hielos son calentados por el sol, y la energía que reciben regresa a la atmósfera como otro tipo de energía, y una vez en ella es retenida por poco tiempo por el vapor de agua, el dióxido de carbono ( $\mathrm{CO})$, el metano (CH4) y otros gases, (clorofluorocarbonos), hidrofluorocarbonos, perfluorocarbobos, óxido nitroso, hexafluoruro de azufre, entre otros). Estos son los gases de efecto invernadero (GEI/GHG's). Se considera que la mayoría de son anteriores a los orígenes del hombre y los únicos que son producto de las acciones antrópicas son los de la familia de los clorofluorocarbonados

Del total de la energía solar que llega al planeta, 30\% es reflejada al espacio exterior; $50 \%$ calienta la superficie terrestre al absorberse por el suelo y metabolizada por las plantas; y 20\% calienta el aire con el que entra en contacto. De la parte absorbida por el suelo, $70 \%$ es reemitida a la atmósfera en forma de radiación infrarroja o calor (energía no visible), debido a la acción de las plantas y al funcionamiento de las cadenas alimenticias. La atmósfera recibe constantemente energía del Sol y también desde la superficie terrestre. (Fondo Verde, 
2016). Esta delgada capa que rodea al planeta equilibra la energía recibida por el Sol y la energía reflejada por la superficie terrestre para mantener un equilibrio en la Tierra.

Sin los gases de efecto invernadero en la atmósfera la temperatura del planeta sería de $-20^{\circ} \mathrm{C}$. Estos gases retienen el calor y favorecen una temperatura promedio global de $15^{\circ} \mathrm{C}$. Ésta es la razón de su denominación, ya que el efecto que producen es similar al de un invernadero: permiten el paso de la energía solar y luego retienen el calor que emite la superficie de la Tierra para mantener un rango de temperatura ideal para la vida.

El cambio climático se manifiesta en un calentamiento global ocasionado por el efecto invernadero. Estos gases se han incrementado sustancialmente en la atmósfera, los que ha generado una rápida retención del calor del planeta. A esto se aúna la dinámica de los sistemas naturales tienen un papel importante, pero las acciones antropogénicas probablemente han actuado como catalizadores aumentando el efecto en el clima a nivel global (Fondo Verde, 2016).

Ante este escenario según (Left, 2011), "no existe una visión comprehensiva y consensuada sobre las causas del cambio climático. Lo anterior, entonces, supone no sólo encontrar mecanismos de mitigación sino buscar también estrategias de adaptación. Ambas estrategias actuarían complementariamente para hacer frente a este fenómeno en situ, e impactaría positivamente en los aspectos conductuales y culturales de todos los miembros de la sociedad.

De igual manera (Retamal, 2011), afirma que la primera estrategia (mitigación), precisa cambios que conduzcan hacia la disminución de los gases de efecto invernadero y de esta manera disminuir o frenar los impactos del cambio climático y, la segunda (adaptación), en la toma de consciencia y realizar acciones tendientes a cambiar cualitativamente el curso del accionar antropogénico, orientando el desarrollo de la economía y la sociedad hacia la sustentabilidad y de esta forma hacer frente a los impactos inevitables del cambio climático.

En ese sentido los órganos rectores de la Organización Meteorológica Mundial y el Programa de las Naciones Unidas para el Medio Ambiente (PNUMA), crearon el Grupo IPCC para orientar y evaluar la información científica sobre el tema y que en 1990 presentaron el primer informe confirmando la amenaza que representaba el cambio climático. Posteriormente en ese mismo año, la segunda Conferencia Mundial sobre el Clima aprobó el tratado mundial a través de la resolución 45/212 en la que se ponía en marcha las negociaciones y compromisos de los países conocidos como las Partes de la Convención (COP), que han realizado reuniones anuales en función del cumplimiento de los acuerdos que ahí se adquieren.

La COP es la Conferencia de las Partes de la Convención Marco de las Naciones Unidas sobre el Cambio Climático(CMNUCC) y la CMP la Conferencia de las Partes en calidad de reunión de las Partes en el Protocolo de Kyoto (CMP).

Uno de los esfuerzos más importantes fue la firma del Protocolo de Kyoto en diciembre de 1997 durante la CP3, celebrada en la ciudad de ese mismo nombre en Japón. Así lo menciona en su informe el (UNFCCC, 2001, pág. 12), “...tras dos años y medio de intensas negociaciones, en la CP3 celebrada en Kyoto (Japón) se aprobó una considerable ampliación de la Convención, en la que se esbozaban compromisos jurídicamente vinculantes de recorte de las emisiones. Era el Protocolo de Kyoto. En él se recogían las normas básicas, pero no se especificaban con detalle cómo deberían aplicarse. Se preveía un proceso independiente y oficial de firma y ratificación por los gobiernos nacionales antes de que pudiera entrar en vigor."

Posteriormente se siguieron una ronda de negociaciones en las siguientes Convención de las partes en función de la aplicación de las normas de Kyoto, la financiación, transferencia tecnológica, y un tercer 
informe del IPCC aportó las pruebas científicas más convincentes mejorando el clima de negociaciones. "Dichas normas se elaboraron con mayor detalle en decisiones posteriores de las CP8, 9 y 10. El Protocolo sólo podía entrar en vigor cuando fuera ratificado al menos por 55 Partes en la Convención, entre ellas un número de países industrializados incluidos en el anexo I que representaran al menos el 55 por ciento de las emisiones de dióxido de carbono de dicho grupo en 1990" (UNFCCC, 2001). El Protocolo entró en vigor el 16 de febrero de 2005.

La Convención fija el objetivo de estabilizar los niveles de emisión de gases de efecto invernadero en un plazo que permita a los ecosistemas adaptarse naturalmente al cambio climático, asegurar la producción de alimentos y permitir el desarrollo económico de manera sostenible (Sistema de la Naciones Unidas del Perú, 2014), y para ello ha dividido a los países en tres grupos principales, en base a sus compromisos, los que se conocen como anexo I, II y III,: los que corresponden al Anexo I son los países industrializados que eran miembros de la Organización de Cooperación y Desarrollo Económico (OCDE) en 1992, más los países con economías en transición (PET), la Federación de Rusia, los Estados Bálticos y varios Estados de Europa Central y oriental, conformada por un total de 43 partes.

La obligación de los países del anexo I es la de "adoptar políticas y medidas relativas al cambio climático con el fin de reducir sus emisiones de gases de efecto invernadero a los niveles de 1990 no más tarde del año 2000". Las Partes incluidas en el anexo II son los países miembros de la OCDE incluidos en anexo I, pero no los PET, estos deben ofrecer recursos financieros para permitir a los países en desarrollo emprender actividades de reducción de las emisiones que les facilite la adaptación a los efectos negativos del cambio climático, a través de la transferencia de tecnologías a los PET y a los países en desarrollo.

Entre 30 Nov y el 11 de diciembre de 2015 se realizó en París la COP21 y la CMP11. La CMNUCC cuenta con 195 países firmantes. Se denomina "Partes" a los países miembros de la Convención. (Sistema de la Naciones Unidas del Perú, 2014).

El principal reto que enfrenta la CMNUCC es lograr un acuerdo estable y eficaz entre los bloques representantes de los países participantes, ya que por un lado los representantes no cuentan con la autoridad para asumir compromisos o no están potestados para hacerlo; de igual manera continuar trabajando en la sensibilización para el cumplimiento de reducción de GEI que han sido definidas a los países desarrollados y los en vías de desarrollo.

La CMNUCC estima que el costo anual para que los países en desarrollo se adapten al cambio climático se encontrará en el orden de los 28-67 mil millones de dólares estadounidenses para el año 2030. Otras estimaciones son incluso mayores. Esto representa un nuevo desafío para que la comunidad internacional asegure niveles adecuados de financiamiento. (Sistema de la Naciones Unidas del Perú, 2014)

Entrelas limitaciones delasiniciativas definanciamiento existentes, destacan dos por su importancia: la severa fragmentación entre distintas iniciativas y los montos limitados de financiamiento que se han entregado a la fecha. Según (Stiftung, 2014), esto no permite a los países crear sinergias entre las metas de adaptación al cambio climático y otras prioridades de desarrollo, lo cual también es un obstáculo para evaluar en qué medida los países desarrollados han cumplido con sus compromisos financieros.

\section{Políticas públicas en el ámbito del cambio climático}

Las políticas públicas son concebidas como "Un conjunto de decisiones y estrategias adoptadas por una autoridad legítima para resolver problemas públicos complejos (Aguilar, 1993). En tal sentido, una política pública es una directriz general que refleja la prioridad y voluntad política del gobierno para modificar una situación determinada. En la práctica, las políticas 
públicas funcionan como instrumentos que permiten al Estado garantizar los derechos humanos y ambientales, vinculando las necesidades sociales de corto plazo con una visión política a mediano y largo plazo, para así eliminar inequidades... (SENPLADES, 2011).

Según un estudio CANLA y la Plataforma Climática Latinoamericana (PCL), dos de las redes climáticas de la Región, afirman que en América Latina existe un aumento significativo en la elaboración de políticas sobre cambio climático, pero que persisten serias deficiencias en su implementación, debido en gran parte a la falta de recursos y de capacidades. (Quintanilla, 2013)

También se aborda la necesidad de formar coaliciones a nivel regional para conseguir el logro de objetivos generales ante los retos climáticos más importantes, tales como integrar una agenda climática para el desarrollo de políticas sectoriales, posicionar las políticas sobre cambio climático en escenarios institucionales centrales, y construir coaliciones sociales y políticas más amplias.

En ese contexto las actuales estrategias en la región necesitan renovarse y ser mejoradas ante la urgencia de crear las condiciones institucionales que permitan atender la problemática que están enfrentando los países ante el cambio climático para tomar decisiones y alcanzar soluciones tangibles.

En un estudio realizado por el PCL, sobre el estado y la calidad de las políticas públicas realizada sobre el cambio climático y el desarrollo en América Latina en 10 países de la región plantea la necesidad de interactuar e involucrar más fuertemente a los actores del sistema político y a otros actores económicos y sociales en el debate sobre la problemática climática, para construir coaliciones políticas y sociales que impulsen y sostengan agendas de desarrollo que efectivamente aborden los desafíos que presenta el cambio climático en los países de la región. (PCL, 2012).
Otro aspecto que destacan es que uno de los principales desafíos de la agenda climática en el sector agropecuario y bosques (temáticas centrales del estudio), "es su falta de integración y articulación con las políticas de desarrollo, de ordenamiento territorial y políticas sectoriales". Lo cual reafirma la necesidad de interactuar e involucrar más fuertemente a los actores de los sistemas políticos, económicos y sociales en el debate de la problemática climática que permita impulsar agendas que aborden los desafíos que presenta el cambio climático en los países en desarrollo.

En este sentido, si el cambio climático como fenómeno ambiental global se requiere de medidas para mitigarlo y enfrentar sus impactos (adaptación) con un enfoque transversal desde todos los ámbitos de la vida: económico, sociales, científicos, tecnológicos, ambientales, educativos, legales entre otros. En ese marco las instituciones necesitan establecer sinergias para atender de manera articulada las estrategias de adaptación en los territorios, para desarrollar políticas para la adaptación al cambio climático dirigidas a reducir la pobreza,

Bajo este contexto (Osorio, 2007), manifiesta que es importante la necesidad que los países adopten Estrategias Nacionales, desde el más alto nivel, para abordar la problemática de manera holística, integral e integrada con una cabal comprensión de la complejidad del fenómeno y sea desde las instancias de Planificación del Estado que se emanen los lineamientos y políticas en los diferentes ámbitos". Como País se cuenta con dos grandes instrumentos que son: el Plan Nacional de Desarrollo y la Estrategia Nacional de Cambio Climático para la mitigación y adaptación.

\section{El Plan Nacional de Desarrollo y la Estrategia Nacional de Cambio Climático en Nicaragua}

El Plan Nacional de Desarrollo y la estrategia nacional y plurianual (2014-2020) del país, concibe la adaptación al cambio climático como una línea de trabajo de alta prioridad; al mismo tiempo refleja que Nicaragua ha 
venido desarrollando estrategias y políticas en materia de energías renovables, así como de los principales desafíos como es el caso de la reforestación del país y la necesidad de financiamiento para implementar las políticas existentes para afincar la frontera agrícola, reforestar el país, conservar las cuencas y adaptarnos al cambio climático (GRUN, 2016).

Los impactos de eventos climáticos recientes como huracanes y sequías sobre los medios de vida de la población nicaragüense han demostrado la elevada vulnerabilidad del país ante el cambio climático. La ONG alemana Germanwatch enlista en su Índice de Largo Plazo de Riesgo Climático (CRI) a tres países centroamericanos entre los 10 países más afectados por el clima entre 1994 y 2013, posicionando a Nicaragua en el cuarto lugar.

En el documento de la Estrategia plurianual describe que la agricultura nicaragüense consta de dos tipos de productivos que coexisten entre sí: por una parte, una producción muy moderna, eficaz, muy productiva, una producción intensiva en insumos y ganadería, basada en el riego de suelos y orientada a las exportaciones; por otra parte, una producción muy tradicional, dependiente del clima, con baja productividad y bajos insumos garantizando la producción de alimentos para el mercado nacional. Esta última no crece mediante la mejora de la productividad, pero extiende los límites de las tierras agrícolas a los bosques húmedos y a los ecosistemas frágiles desde un punto de vista ambiental. (GRUN, 2016).

Además refiere que como consecuencia de ello, muchos ecosistemas de Nicaragua están degradados, con tasas de elevadas a muy elevadas de erosión del suelo, bosques que desaparecen rápidamente, una biodiversidad en peligro y una menor capacidad de apoyo a la agricultura, lo que a su vez pone en peligro la seguridad alimentaria.

La situación de que la economía del país se base principalmente en la explotación de los recursos naturales la hace especialmente vulnerable a la degradación del suelo, el agua y los bosques, agravada por los efectos del cambio climático, con graves repercusiones considerables en la economía y el desarrollo de Nicaragua. Con el fin de lograr una mayor resiliencia, especialmente en las zonas rurales, deben reforzarse las medidas y los presupuestos del Gobierno, y es necesario fomentar la comprensión de conceptos técnicos relacionados con el cambio climático.

Para lo que es particularmente importante que, para la adaptación al cambio climático, se requiere de contar con un sistema de información moderno y accesible a todos; contar con un alto nivel de concientización a todos los niveles sobre el tema; definir una política específica sobre el tema del cambio climático; y establecer una visión de administración del riesgo con especial énfasis en el uso de tecnologías que permitan predecir eventualidades y planificar acciones concretas. Como País se deben fomentar |medidas con suficientes co beneficios para contribuir no solo a la lucha contra el cambio climático, sino también a la reducción de la pobreza y otras metas de desarrollo.

A nivel internacional Nicaragua deberá evidenciar el valor de sus ecosistemas para la captura y almacenamiento de carbono y demostrar sus contribuciones en la reducción de la emisión de gases de efecto invernadero. Esto implica trabajar en mecanismos más sencillos para que las comunidades puedan acceder a los recursos disponibles y participar en el mercado de pagos por servicios ambientales y evitar la severa deforestación que están sufriendo las zonas boscosas del país (GRUN, 2016).

De igual manera se reafirma la necesidad de establecer alianzas que puedan generar procesos de debate orientadas a la sensibilización de todos los territorios. En el país, estas alianzas son posibles por el proceso de concertación que ha impulsado el gobierno que puede facilitar llevar propuestas más robustas y concretas sobre la mitigación o posicionamiento internacional. Estas alianzas pueden facilitar el apoyo para el manejo 
de datos climáticos reales y divulgar las experiencias exitosas que han impulsado acciones novedosas de reducción y uso de combustibles limpios en sus procesos productivos.

El cambio climático se ha convertido en una prioridad, así se refleja en la Estrategia Regional de Cambio Climático aprobada en 2010. En términos de integración de las cuestiones medioambientales, el índice de degradación supera, con creces, la capacidad de los Estados de AC para responder con prontitud. De igual manera se Se han formulado algunas políticas regionales pertinentes y se han establecido algunas prioridades estratégicas, pero los países están todavía en el proceso de su integración a escala nacional. Según un informe sobre la situación presente y futura de la biodiversidad en Centroamérica, la pérdida de biodiversidad en la región asciende a un promedio del $52 \%$. Esta pérdida se debe principalmente a la utilización del suelo, las infraestructuras viarias, la fragmentación de los ecosistemas naturales y el cambio climático (GRUN, 2010).

La Estrategia plantea claramente que el cambio climático puede tener repercusiones considerables en la economía y el desarrollo de Nicaragua. A la vez plantea la necesidad de que, para lograr una mayor resiliencia, especialmente en las zonas rurales, deben reforzase las medidas y los presupuestos del Gobierno, y es necesario fomentar la comprensión de conceptos técnicos relacionados con el cambio climático.

Otros elementos sumamente importantes son los roles de la participación ciudadana y las universidades en la investigación para aportar con la información que contribuiría a dar un enfoque más concreto soluciones posibles en los territorios donde inciden, tomando en cuenta la alta calidad profesional en este campo, lo cual podría trascender del enfoque estrictamente ambiental, sino más bien vincularlo o transversalizarlo con otras problemáticas de manera que se involucran todos con una visión integral en las acciones. Estos enfoques son la seguridad alimentaria, los precios del mercado, las políticas públicas e iniciativas económicas apoyadas por el gobierno con la población y otros programas.

De igual manera, contar con una política institucional sobre la adaptación al cambio climático debe conciliar esfuerzos en considerar el acceso a recursos que permitan la actuación integral en el tema; lo cual posibilita el aprovechamiento óptimo de los recursos asignados a la implementación de esas políticas.

\section{Política institucional de adaptación al cambio climático}

Con esta base, se puede comprender la necesidad de contar con instrumentos de política, normativos y técnicos para facilitar que "los distintos sectores y actores de nuestra sociedad, incorporen la temática de adaptación al cambio climático en sus lineamientos, objetivos o metas, con el propósito de construir la institucionalidad requerida para que dicho proceso se desarrolle de manera eficiente" (MINAM, 2015).

La urgente necesidad para enfrentar el cambio climático exige que el aprendizaje continuo se constituye en un elemento clave para lograr incidir en la solución de los problemas ambientales, para contribuir a la construir de una cultura ambiental que aporten a la protección de los recursos naturales y del ambiente, y puede ser posible cuando el nivel de atención del problema se jerarquiza en una política institucional que conduzca los esfuerzos y los impactos sean mas contundentes.

Anivel global, la educación en cambio climático implica también nuevos desafíos que van desde contar con una visión holística, integradora de diferentes disciplinas, hasta promover la participación, el intercambio de información y de experiencias; la investigación en tecnologías ambientales al servicio de todos los países; la difusión de información e investigación que deben abordar no sólo aspectos ambientales o ecológicos, sino también sociales, culturales, económicos y políticos.

En ese sentido la UNAN Managua, en su misión se plantea "Formar profesionales y técnicos integrales 
desde y con una concepción científica y humanista del mundo, capaces de interpretar los fenómenos sociales y naturales con un sentido crítico, reflexivo y propositivo, para que contribuyan al desarrollo social, por medio de un modelo educativo centrado en las personas,... todo ello en un marco de cooperación genuina, equidad, compromiso y justicia social y en armonía con el medio ambiente (UNAN-Managua, 2016).

Este rol es afirmado por (Lopez, s.f.), refiere que "frente a los retos que impone el cambio climático, es necesario crear resistencia al mismo, lo que pasa necesariamente por un proceso sostenido de adaptación, que implica la puesta en práctica de medidas, tanto en el marco normativo, como también procesos de empoderamiento de la sociedad, donde el factor educativo será esencial, para la creación de una conciencia individual y colectiva, para que las respuestas sean integrales, tanto en lo social como en lo económico."

También destaca la necesidad de capacitar a la población en general y a los productores en particular, es determinante, para establecer mecanismos de adaptación al cambio climático, contribuirá sobremanera para que los Estados cuenten con "políticas públicas en torno a este fenómeno y sus efectos, además de contribuir a través de programas transversales mediante los cuales se logre profundizar en investigaciones sobre protección de ecosistemas y utilización de energías alternativas, entre otras, para disminuir y controlar las emisiones de gases de efecto invernadero.

Aún hay mucho por hacer pero lo claro es que si la universidad cuenta con un instrumento institucional logrará concentrar y organizar el esfuerzo de todas sus facultades, fortalecer las sinergias con todos los sectores a todos los niveles a fin de crear redes importantes que permitan la incidencia y compartir información, acceder a recursos para poder contribuir de manera significativa en la adaptación al cambio climático. La atención a los desafíos del cambio climático requiere también una visión estratégica de mediano y largo plazo, aspecto en el que la UNAN Managua es un referente importante.

\section{CONCLUSIONES}

La adaptación al cambio climático es uno de los retos más grandes en la armonización de los marcos legales a todos los niveles que permitan desarrollar acciones de impacto para disminuir la vulnerabilidad y la pobreza de Nicaragua; por lo que es sumamente urgente la construcción de una política institucional en la UNAN Managua que facilite contribuir de manera importante. Una política institucional permitirá trabajar en el fortalecimiento del campo de la investigación, la transformación curricular integrando el cambio climático como eje transversal curricular en todas las carreras de manera que el profesional que egrese tenga una visión de responsabilidad social y ambiental. Así como articular las capacidades humanas y materiales en función de la adaptación al cambio climático. De igual manera construir alternativas de adaptación y superar los impactos.

Con base en estos planteamientos el rol de las universidadesessumamenteindispensableconsiderando que se puede contribuir con la información científica a la base de datos de cambio climático del país, crear capacidades en materia ambiental a los estudiantes y personal docente; así como hacer incidencia en que la transformación curricular integre el eje transversal de cambio climático en las diversas carreras que oferta.

Este proceso también contribuye al modelo de desarrollo que impulsa el Gobierno de Reconstrucción Nacional del País, orientado a la sustentabilidad y el bienestar de las grandes mayorías que conduce a la revisión de los patrones de producción y consumo hacia un modelo socioeconómico más solidario, humanista y respetuoso de la naturaleza y de las generaciones futuras. 


\section{REFERENCIAS BIBLIOGRÁFICAS}

Fondo Verde. (2016). Bases Teóricas, Racionalidad Ambiental, Causas y Evidencias y Escenarios del Cambio Climático. España: Ambiental.

GRUN. (2010). Estrategia Nacional y Ambiental de CambioClimático 2010-2015. Managua, Nicaragua. GRUN. (2016). Estrategia Nacional y Programa Indicativo Plurianual 2014-2020. Managua.

IPCC. (2000). Escenario de Emisiones. España.

IPCC. (2007). Cambio climáticoInforme de sintesis. Contribución de los Grupos de trabajo I, II y III al Cuarto Informe de. Ginebra, Suiza.

Left, E. (2011). Sustentabilidad y racionalidad ambiental: hacia otro programa de sociologia. Revista Mexicana de Sociología, 5-46.

Londoño, O. L. (2014). Guía para construir estados del arte. Bogotá.

Lopez, E. (s.f.). Cambio climático y su impacto en la economía y la sociedad nicaraguense. Obtenido de http://www.farem.unan.edu.ni/ redcambioclimatico/docs/CC_y_su_impacto_en_ CA.pdf

Lugo Marín, D. D. (2015). Bases Teóricas, Racionalidad ambiental, Causas y Evidencias y Escenarios del Cambio Climático. España.

Martinez, L. (1999). “¿Qué significa construir un estado del arte desde una perspectiva hermenéutica? Revista Criterios, 13-20.

Milán, J. (26 de Noviembre de 2016). UCC. Obtenido de http://www.ucc.edu.ni/noticias-anterioresucc/1776-informan-sobre-impacto-del-cambioclimatico-en-nicaragua

MINAM. (2015). Lineamientos para la incorporación de la adaptación al cambio climático en la Universidad Peruana. Perú.

OMM. (2009). El estado de la investigación polar. Génova.

Osorio, A. E. (2007). Cambio Climático y Políticas Públicas. Encuentro Internacional Clima Latino.
Quayaquil, Ecuador.

PCL. (Noviembre de 2012). Plataforma Climática Latinoamericana. Obtenido de http://www. ambienteycomercio.org/publicacion-politicaspublicas-y-cambio-climatico-en-los-sectoresagricola-y-forestal-de-america-latina/

Quintanilla, V. (19 de Noviembre de 2013). AIDA. Obtenido de http://www.aida-americas.org/es/ blog/pol\%C3\%ADticas-p\%C3\%BAblicas-paracambio-clim $\% \mathrm{C} 3 \%$ A 1 tico-en-am $\% \mathrm{C} 3 \%$ A9ricalatina

Retamal, e. a. (2011). Percepción al cambio climático y a la gestión del agua: aportes. Ambiente y Sociedad, 175-194.

SENPLADES. (2011). Guía para la formulación de políticas públicas sectoriales. Quito.

Sistema de la Naciones Unidas del Perú. (11 de Marzo de 2014). ¿Que es la COP? Obtenido de http://onu. org.pe/cop-20/que-es-la-cop-20/

Stiftung, H. B. (Diciembre de 2014). Climate Funds Update. Obtenido de https://www.odi.org/sites/ odi.org.uk/files/odi-assets/publications-opinionfiles/9315.pdf

UNAN. (27 de Noviembre de 2016). UNAN Managua,. Obtenido de http://www.unan.edu.ni/index.php/ proyecto-institucional/

UNFCC. (s.f.). United Nations Framework Convention on Climate Change. Obtenido de http://unfccc.int/ portal_espanol/informacion_basica/protocolo_de_ kyoto/items/6215.php

UNFCCC.(Noviembre de 2001). Guía dela Convención sobre el Cambio Climático y el Protocolo de Kyoto. Obtenido de http://unfccc.int/resource/docs/ publications/unitingonclimate_spa.pdf

UNFCCC. (28 de Junio de 2017). La Convención de las Partes. Obtenido de Organos de la Convención: https://unfecc.int/portal_espanol/informacion_ basica/la_convencion/estructura/organos/ items/6209txt.php 